D.O.I.: $10.3895 / \mathrm{S} 1808-04482011000300012$

\title{
UMA APLICAÇÃO PRÁTICA DA GESTÃO DO CONHECIMENTO NO FRONT OFFICE DE UM CONTACT CENTER
}

\section{A PRACTICAL APPLICATION OF KNOWLEDGE MANAGEMENT TO THE FRONT OFFICE OF A CONTACT CENTER}

\author{
Samuel Ribeiro Tavares ${ }^{1}$; Milton Vieira Júnior ${ }^{2}$ \\ ${ }^{1}$ Universidade Nove de Julho - UNINOVE - São Paulo - Brasil \\ samueltavares@yahoo.com \\ ${ }^{2}$ Universidade Nove de Julho - UNINOVE - São Paulo - Brasil \\ mvieirajr@ig.com.br
}

\begin{abstract}
Resumo
Este trabalho teve por objetivo desenvolver e testar uma proposta baseada na gestão do conhecimento organizacional, que permitisse a uma operação de contact center, ao mesmo tempo, colher os beneficios da abordagem empowerment e minimizar os riscos dela decorrentes. Em uma pesquisa experimental, intervencionista, com um componente de orientação crítica, estudou-se o caso de uma destas operações em São Paulo. À luz dos resultados obtidos, é possível afirmar, sem, contudo, qualquer pretensão de generalização, que a gestão do conhecimento pode contribuir para uma efetiva implementação da abordagem empowerment em contact centers.
\end{abstract}

Palavras-chave: gestão do conhecimento; empowerment; contact center; operações.

\section{Introdução}

Entre as várias ações para unir foco estratégico e capacidade de execução, as empresas lançam mão de centrais de operações de serviço para interação multimídia direta, dinâmica e diferenciada com os consumidores de suas ofertas: os contact centers (BERGEVIN, 2005), que, ao lhes possibilitarem interagir com seus clientes de forma imediata, monitorando, entendendo e (re)agindo em função de suas percepções, são capazes de contribuir para uma melhor gestão do ciclo de vida dos clientes pelas organizações (CURRY, 2000).

Para tanto, em sua configuração completa, os contact centers incorporam cinco áreas de atividade, voltadas para a cobertura de todas as etapas do processo de venda, de modo a expandir ao máximo o ciclo de vida dos clientes junto à organização (SHARP, 2003): marketing, relativa a transações de venda, abrangendo informações sobre o produto ou serviço, auxílio aos clientes na decisão quanto à melhor opção e negociação de condições preços; fulfillment, relativa a transações operacionais, abrangendo entrega ou reparo de produtos físicos, (re)disponibilização de serviços aos 
clientes, e/ou processamento de dados (e.g. pedidos de venda, pesquisas de campo); customer care, relativa a transações de apoio ou suporte aos clientes, abrangendo ações de atendimento para tratamento às suas dúvidas, solicitações ou reclamações referentes a produtos ou serviços; collection, relativa a transações de pagamento pela aquisição e/ou utilização de produtos ou serviços de uma empresa, abrangendo ações de negociação para (re)financiamento de dívidas vencidas; retention, relativa a transações de retenção de clientes que queiram devolver produtos/cancelar serviços, abrangendo ações de negociação que sejam a última linha-de-defesa para manutenção de sua relação com a empresa.

Assim como em outras atividades empresariais, um dos grandes desafios na gestão de contact centers é encontrar o melhor modo de juntar os blocos de construção organizacional num projeto de trabalho que, efetivamente, alinhe pessoas, processos e tecnologia (HICKEY; LAKE, 2003), e, normalmente, estas operações possuem uma estrutura composta por um interactive voice response (computador que interage com clientes), um front office (pessoas que interagem com clientes) e um back office (pessoas que processam as solicitações dos clientes ao front office) (JOHNSTON; CLARK, 2002), visando, respectivamente, possibilitar autosserviço aos clientes; oferecer um toque humano nas interações com clientes; e retirar atividades mais complexas e financeiramente sensíveis do front office, reservando-lhe mais tempo a ações de relacionamento com clientes (CORRÊA; CAON, 2002).

Contudo, de maneira geral, os contact centers não têm cumprido a contento seu papel de, correspondendo às expectativas de seus clientes, colaborar para sua fidelização, garantindo, com práticas efetivas, o retorno sobre o investimento das empresas (MADRUGA, 2006): “ ... na tentativa de alterar o endereço onde recebe a conta de seu celular, o empresário ligou para o call center da operadora da qual é cliente ... ficou vinte minutos falando com a atendente ... no mês seguinte, a conta foi para o endereço antigo ... já a economista, com muito custo, conseguiu agendar um técnico ... a atendente disse que, se ela estivese em casa, a visita não seria cobrada, caso contrário, cobrariam ... ela desmarcou um compromisso, e ninguém apareceu ... só quatro dias após o combinado ..." (PONTES, 2009). Um ano após o início da vigência do Decreto 6.523, que regulamenta os serviços de atendimento ao consumidor, embora tenha havido melhoria no desempenho das empresas, elas continuam muito longe de cumprir totalmente as novas regras (VIALLI; CRUZ, 2009): só em São Paulo, neste período, o PROCON (Procuradoria de Proteção e Defesa do Consumidor) multou 43 empresas, num valor total de R\$35 milhões, e, entre as principais infratoras, encontram-se bancos, empresas de planos de saúde e companhias de telefonia (BIAZZI, 2009); no topo do ranking de queixas, estão reclamações quanto ao tempo de espera para ser atendido, reclamações de ligação interrompida antes de o atendimento ser concluído, e reclamações de falta de preparo dos atendentes para conduzir os problemas à solução (PONTES, 
Embora busquem a capacidade de agir, i.e. união entre competência e comprometimento (TOMASKO, 1994), a extensa maioria das operações de contact center constitui um contingente despreparado, inchado e burocrático, cuja baixa eficiência operacional dificulta a eficácia mercadológica (FITZSIMMONS; FITZSIMMONS, 2007). Sob uma perspectiva ampla, estas operações se posicionam ao longo de um contínuo no qual seu projeto de trabalho pode ir de uma força-de-trabalho robotizada sob rígida hierarquia a um time com quase irrestrito poder para tomar decisões (NICKERSON, 2004; WALTON, 2006): num extremo, a abordagem "linha-de-produção", adaptada das operações de manufatura para as operações de serviço, baseia-se nas práticas de dividir o trabalho, simplificar as tarefas e fornecer pouco poder de decisão para os funcionários (LEVITT, 1972, 1976) - apesar de propiciar alto controle sobre as atividades, o foco restrito e o pouco poder de decisão concedidos aos operadores tornam lenta a ação empresarial e frustram expectativas de clientes, por soluções certas e rápidas, e de funcionários, por crescimento e reconhecimento (LAWLER, 1998; ALSOP et al., 2006); no outro extremo, a abordagem "empowerment", utilizada a partir dos anos 90 tanto em operações de manufatura como de serviço, baseia-se no desenvolvimento de funcionários com alta autonomia e grande motivação para tratar situações diversas de modo diverso (GALBRAITH; LAWLER, 1995; ALSOP et al., 2006) embora apreciada por clientes, pelo tratamento diferenciado recebido, e por operadores, pela possibilidade de atuação autônoma, esta abordagem, geralmente empírica, tende a gerar custos mais altos e níveis de serviço inconsistentes, tanto quantitativa como qualitativamente (ARGYRIS, 1998; BATESON; HOFFMAN, 2001; LELAND; BAILEY, 2006). Em geral, os contact centers não oferecem um ambiente favorável à efetiva promoção da capacidade de agir dos operadores, por não gerirem adequadamente seu capital intelectual (DIJULIUS, 2008), desconhecendo como obter, compartilhar e usar seu conhecimento organizacional (LUCAS, 2008).

Trabalhando com contact centers há algum tempo, estes pesquisadores foram convidados a desenvolver e testar uma proposta que permitisse a uma destas operações, ao mesmo tempo, colher os benefícios da abordagem empowerment e minimizar os riscos dela decorrentes, e, por um lado, cientes de estudos que mostram que a gestão do conhecimento é capaz de promover competência e comprometimento nas empresas (DAVENPORT et al., 2002), ao prover capacidade de execução, base da competência, e foco estratégico, base do comprometimento (LEILBOLD et al., 2005); e, por outro lado, entendendo ciência como um conjunto de atitudes e atividades racionais dirigidas ao sistemático conhecimento do mundo, com objetivos determinados, capaz de serem submetidos à verificação (TRUJILLO FERRARI, 1974; MARCONI; LAKATOS, 2006), aceitaram o desafio: este artigo sintetiza os principais pontos deste empreendimento, que buscou responder à pergunta "A gestão do conhecimento pode ser usada para permitir que os contact centers, efetivamente, 
utilizem a abordagem empowerment?".

\section{Desenvolvimento}

Com duração de 9 meses, esta pesquisa teve por objetivo final, viabilizado por meio de cinco objetivos intermediários, verificar se a gestão do conhecimento poderia contribuir para uma efetiva implementação da abordagem empowerment em contact centers:

- o objetivo intermediário 1 foi o de revisar a literatura especializada, em busca de informações relevantes para a proposição de um modelo teórico baseado na gestão do conhecimento para aplicação da abordagem empowerment;

- o objetivo intermediário 2 foi o de conhecer a realidade do contact center no qual se realizaria a pesquisa, de modo a proporcionar o alinhamento de objetivos, recursos, metodologia e expectativas, e garantir a adequada execução do estudo;

- o objetivo intermediário 3 foi o de desenvolver um roteiro para aplicação do modelo teórico proposto, a fim de assegurar que a mudança organizacional por ele trazida fosse adequadamente gerida, de modo a possibilitar sua apropriação pelos envolvidos;

- o objetivo intermediário 4 foi o de usar o roteiro para aplicação do modelo teórico proposto na identificação e implementação de ações, através das quais se poderia avaliar o impacto da proposta sobre o desempenho do contact center;

- o objetivo intermediário 5 foi o de avaliar o resultado das ações implementadas, a fim de que se pudesse conhecer o efeito da aplicação do modelo teórico proposto sobre o desempenho do contact center e o papel da gestão do conhecimento neste processo.

Quanto aos fins, ao estudar a influência de uma abordagem com base na gestão do conhecimento para aumento da capacidade de agir dos funcionários em uma empresa, esta foi uma pesquisa experimental (GIL, 1989); quanto aos meios, ao interferir para modificar a realidade estudada, não só propondo uma resolução para seus problemas, mas tentando resolvê-los de maneira efetiva e participativa, este estudo constituiu uma investigação intervencionista (VERGARA, 1998); quanto ao posicionamento frente ao conhecimento, ao encarar o desempenho empresarial como um imperativo em torno do qual todo conhecimento ou prática deve ser gerado, este trabalho apresentou, stricto sensu, uma orientação não crítica (DAVEL; ALCADIPANI, 2003), contudo, ao, explicitamente, indicar que as pessoas que constituem as organizações devem aprender permanentemente umas com as outras, com seus clientes, fornecedores e mercados, este estudo trouxe em si um componente crítico (VERGARA; BRANCO, 2001), na medida em que não só admitiu a possibilidade, como buscou contribuir para estimular o desenvolvimento de modelos gerenciais que tragam menos dominação e exclusão, rejeitando ações opressivas, percebidas como 
inúteis nas empresas no mundo contemporâneo.

Como método de abordagem, este estudo utilizou o método hipotético-dedutivo, que, ao perceber lacunas de conhecimento, gera hipóteses e, por inferência dedutiva, testa a predição da ocorrência de fenômenos/fatos por elas abrangidos (POPPER, 1975a, 1975b); como método de procedimento, ele se embasou no estruturalismo, que analisa um acontecimento concreto, gera um modelo abstrato que retrata o objeto estudado, e volta ao concreto, numa realidade estruturada da experiência dos sujeitos sociais (KAUFMANN, 1977); como método de investigação, ele lançou mão da pesquisa-ação, no qual pesquisadores e sujeitos representativos do problema decidem, empiricamente, envolver-se de modo cooperativo/participativo na tentativa de resolver um problema (THIOLLENT, 1997).

Dentre as técnicas de coleta de dados, este estudo utilizou pesquisa documental, observação participante, entrevistas (estruturadas e não-estruturadas não-dirigidas) e questionários (perguntas abertas, fechadas e de estimação com escala Lickert) (MARCONI; LAKATOS, 1990); e, dentre as técnicas de análise de dados, este estudo utilizou tanto a abordagem de tratamento quantitativo, como a abordagem de tratamento qualitativo (MARCONI; LAKATOS, 1990).

\subsection{Objetivo intermediário 1 - um modelo teórico}

Empowerment consiste em preparar e encorajar as pessoas para agirem (PFEIFFER; DUNLOP, 1990), transformando informações em ações que contribuam para a solução de problemas e a inovação em seu local de trabalho (CUNNINGHAM; HYMAN, 1999), não como um fim em si mesmo, mas como um meio de se concretizar uma visão (GINNODO, 1992). Trata-se de um sentimento de empenho com o trabalho, trazido à tona pela habilidade de se tomar decisões, ser responsável, ser avaliado por resultados, e, principalmente, ser reconhecido como alguém que pensa e contribui (COLE, 1995). Sua argumentação básica, suportada por várias pesquisas (ALSOP et al., 2006; SIMONS, 2008), é que a motivação individual aumenta e os resultados melhoram quando as pessoas estão aptas a e têm estímulo para decidir sobre seu trabalho e suas metas (DESSLER, 1999).

Os programas de empowerment apresentam quatro dimensões - visão compartilhada (objetivos e expectativas), estrutura organizacional (níveis de responsabilidade e apoio), aprendizado (acesso ao e manipulação do conhecimento) e reconhecimento (contribuições e resultados) - (HERRENKOHL et al.,1999), e necessitam de um contexto organizacional capaz de fornecer know-how (capacidade de execução) e know-why (foco estratégico) aos funcionários (POTTERFIELD, 1999): os gestores só conseguem desenvolver a capacidade de agir dos funcionários quando compartilham informação, estimulam autonomia e trabalho em equipe, oferecem treinamento relevante, e recompensam seus colaboradores pelos riscos e iniciativas deles 
esperados (QUINN; SPREITZER, 1999).

Desde o fim dos anos 80, várias empresas começaram a perceber que, mais que um componente casual ou inconsciente, o conhecimento organizacional constitui fator crítico de sucesso, capaz de influenciar lucros presentes e futuros (DAVENPORT, 2005), e, explícita e sistematicamente, passaram a identificar os conhecimentos vitais a seus negócios e os processos básicos de obtenção, organização, difusão e exploração a eles ligados (SKYRME, 1999), e a oferecer maior valor a seus stakeholders (ALVARENGA NETO, 2008), iniciando a chamada gestão do conhecimento (NONAKA, 1991): a competência em mapear e aproveitar o conhecimento organizacional se converteu, assim, em poderosa arma competitiva (SUFFERT, 2008), e redefiniu a atividade empresarial de saber fazer coisas para saber fazer coisas novas, de modo eficiente e eficaz (DAVENPORT; PRUSAK, 1998).

Efetivamente capaz de fornecer tanto know-how como know-why, a gestão do conhecimento constitui uma alternativa viável para promover o empowerment nas organizações empresariais (DAVENPORT, 2005; FIGUEIREDO, 2005): estrategicamente, ela propicia um modelo de gestão baseado na aprendizagem organizacional voltada para o aumento do valor da empresa, por meio do desenvolvimento da capacidade das pessoas de sentirem e reagirem efetivamente às mudanças (DAVENPORT; PRUSAK, 1998); taticamente, ela possibilita o acesso dos funcionários a dados e informações que, influenciando seus conhecimentos, habilidades e atitudes, lhes permitem identificar soluções inovadoras que aumentam a eficácia mercadológica da empresa (SKYRME, 1999); operacionalmente, o compartilhamento de dados e informações favorece a integração de pessoas, processos e tecnologia na entrega de soluções personalizadas que equilibram os aspectos quantitativos e qualitativos da eficiência operacional (SVEIBY, 1998).

Refletindo sobre estas informações, estes pesquisadores acreditaram haver uma possível relação entre as quatro dimensões do empowerment e os quatro processos básicos associados à gestão do conhecimento, e, buscando explicitá-la, aprofundaram sua investigação:

- a dimensão visão compartilhada se refere à definição por uma empresa de seus valores e metas fundamentais como condição básica para que seus funcionários possuam foco, energia e confiança na tomada de decisões individuais e/ou conjuntas (COLLINS; PORRAS, 1998); o processo básico obtenção do conhecimento organizacional diz respeito à formulação de uma política estratégica corporativa que possibilite o desenvolvimento e aplicação do conhecimento na empresa como um todo (LARA, 2004);

- a dimensão estrutura organizacional se refere à definição pelas empresas dos níveis de obrigação nas tomadas de decisão e ao estabelecimento de mecanismos de suporte às ações adotadas, e à utilização da tecnologia da informação para estimular o trabalho colaborativo 
(MALONE, 2004); o processo básico organização do conhecimento organizacional diz respeito à definição de responsabilidades e tarefas voltadas à identificação, mapeamento e reunião dos ativos de conhecimento da empresa de modo a facilitar seu compartilhamento (JAMIL, 2006);

- a dimensão aprendizado se refere à busca pelos funcionários de conhecimento para a melhoria de seu desempenho e reconhecimento individual e/ou coletivo, e à criação de oportunidades de um aprendizado organizacional efetivo (BLANCHARD et al., 2001); o processo básico difusão do conhecimento organizacional diz respeito à definição e implementação de estratégias técnicas e motivacionais para aumentar e tornar relevantes os conhecimentos particulares (pessoas, equipes e/ou áreas) e coletivo da empresa (SANTIAGO JR., 2004);

- a dimensão reconhecimento se refere à compensação (financeira e/ou não financeira) pela empresa ante o desempenho e contribuições (individuais e/ou coletivos) de seus funcionários, mostrando-lhes que eles estão sendo ouvidos, apreciados e apoiados (JAFFE; SCOTT, 1992); o processo básico exploração do conhecimento organizacional diz respeito ao acompanhamento da melhoria diária da organização em função do uso e o desenvolvimento do conhecimento frente aos objetivos propostos e no reconhecimento de seus contribuintes (CARBONE et al., 2006).

Analisando-se individualmente as dimensões e os processos básicos, em busca de sua coerência interna, percebeu-se que ambos constituíam funções administrativas gerais planejamento, i.e definição de objetivos e como atingi-los; organização, i.e. atribuição de tarefas, recursos e responsabilidades; direção, i.e. influenciação para alcance dos objetivos; e controle, i.e. monitoração do e recompensa pelo desempenho (DAFT, 2007) - capazes de viabilizar o empowerment e a gestão do conhecimento, respectivamente:

- na função planejamento, a visão compartilhada dá intento ao empowerment ante o negócio e serve de estímulo referencial à competência de tomar decisão; a política estratégica corporativa orienta a definição e aplicação geral dos conhecimentos relevantes ao negócio;

- na função organização, a estrutura organizacional indica os processos principais e os deveres básicos no empowerment; a organização do conhecimento localiza os conhecimentos-chave da empresa e aponta as pessoas com quem podem ser obtidos, discutidos e atualizados;

- na função direção, a aprendizagem organizacional age como infraestrutura técnica e motivacional para o empowerment; a difusão do conhecimento empresarial constitui infraestrutura técnica e motivacional para formação e uso do capital intelectual das empresas;

- na função controle, acompanhamento de e recompensas por contribuições e desempenho suportam e incentivam o empowerment; acompanhamento uso e renovação relevantes do conhecimento, com apreço às pessoas, suporta e incentiva a gestão do conhecimento. 
Analisando-se em conjunto as dimensões e os processos básicos, em busca de sua possível inter-relação, inferiu-se que os processos básicos poderiam maximizar as chances de sucesso do empowerment, ao cobrirem aspectos essenciais a qualquer ação empresarial - negócio, i.e. propósito e competências essenciais da empresa; processos, i.e. processos-chaves de negócio e papéis e responsabilidades envolvidos; tecnologia, i.e. infraestrutura à operação, comunicação e gestão empresarial; pessoas, i.e. suporte e incentivo aos envolvidos - que potencializam a possibilidade de alcance de metas organizacionais (IVES; GERSTING, 1998):

- no aspecto negócio, uma política estratégica corporativa que orientasse a definição e aplicação geral de conhecimentos relevantes ao negócio poderia contribuir para o foco, energia e confiança dos funcionários na tomada de decisões individuais e/ou conjuntas;

- no aspecto processos, a localização dos conhecimentos-chave da empresa e das pessoas com quem poderiam ser obtidos, discutidos e atualizados poderia contribuir para definir processos e deveres das pessoas, e uso da tecnologia de informação, nas tomadas de decisão;

- no aspecto tecnologia, a difusão do conhecimento empresarial, infraestrutura para criação e uso do capital intelectual nas empresas, poderia subsidiar a busca de conhecimento pelos funcionários para melhor desempenho e maior reconhecimento individual e/ou coletivo;

- no aspecto pessoas, a monitoração e reconhecimento da empresa pelo uso e renovação do conhecimento pelos funcionários poderia instrumentalizar ações de compensação (financeira e/ou não financeira) por desempenho e contribuições (individuais e/ou coletivos).

Como resultado destes estudos, ao longo de 3 semanas, obteve-se um modelo teórico (Figura 1) baseado na gestão do conhecimento para maximização dos benefícios e minimização dos riscos decorrentes da aplicação da abordagem empowerment.

Figura 1: Modelo teórico

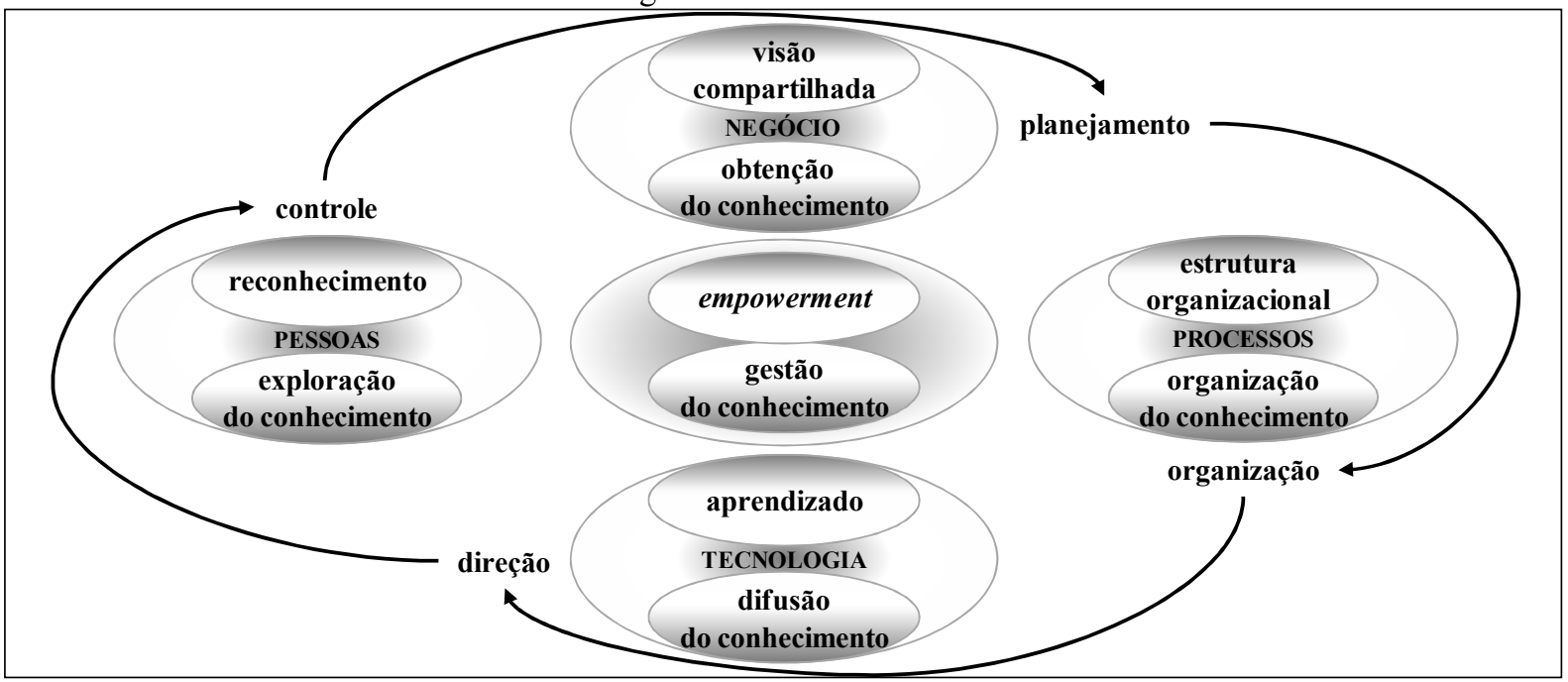

Fonte: Baseado em HERRENKOHL et al. (1999); SKYRME (1999); DAFT (2007); IVES; GERSTING (1998) 


\subsection{Objetivo intermediário 2 - a realidade do contact center estudado}

Ao longo de 1 semana, estes pesquisadores conversaram com os representantes do contact center que solicitou a realização desta pesquisa - parte de uma empresa de cartões de crédito em São Paulo - com o objetivo de conhecer sua realidade.

Embora o contact center contasse com as cinco áreas de cobertura do processo de venda (vide 1), este estudo, por decisão de seus representantes, se limitaria ao customer care (vide 1), formado por um interactive voice response (vide 1), um front office (vide 1), um back office (vide 1) e um time de apoio à gestão:

- o interactive voice response, apesar de fundamental a estas operações, em sua procura por aperfeiçoar e inovar seu relacionamento com clientes e parceiros, não seria contemplado neste estudo, que tinha por foco a autonomia decisória dos funcionários nestas operações;

- o front office era formado por 12 equipes de atendimento, cada equipe composta por 25 operadores (num total de 300) e ligadas a 1 supervisor(a) (num total de 12), todas elas sob responsabilidade de 1 gerente;

- o back office era formado por 2 equipes de processamento, cada equipe composta por 25 analistas (num total de 50) e ligadas a 1 supervisor(a) (num total de 2), ambas sob responsabilidade de 1 gerente;

- o time de apoio à gestão era formado por 1 equipe de planejamento ( 3 consultores), 1 equipe de seleção ( 2 selecionadores), 1 equipe de treinamento (com 5 instrutores) e 1 equipe de controle de qualidade (com 6 monitores), ligados a 2 supervisores.

A questão fundamental para os representantes da empresa era a percepção de seus clientes de que o front office, embora cortês, não oferecia soluções imediatas a várias de suas solicitações, repassadas a pessoas (supervisores) ou área (back office) "responsáveis": sua decisão de envolver estes pesquisadores visava tentar reverter esta situação, fazendo com que o front office conseguisse, com grande deferência, converter escolhas fundamentadas em ações oportunas, em contatos diretos, dinâmicos e únicos com os clientes.

Contanto que o nome da empresa não fosse, de modo algum, mencionado, seus representantes concordaram com o registro dos principais pontos desta experiência, sob a forma de um artigo científico, e com sua divulgação no meio acadêmico-empresarial.

\subsection{Objetivo intermediário 3 - um roteiro para aplicação do modelo teórico proposto}

Acreditando que os sujeitos de pesquisa devem participar da proposição, implantação e avaliação de qualquer intervenção em sua realidade (vide 2), estes pesquisadores, ao longo de 2 semanas, definiram com os representantes da empresa os participantes-chaves do estudo, i.e. 
pessoas oriundas de áreas e níveis hierárquicos heterogêneos na empresa, e possuidoras de perfil técnico-comportamental que lhes possibilitassem entender e adequar o modelo teórico proposto à realidade da organização, e de conduzir sua adequada aplicação (VERGARA, 2005): foram escolhidos 8 colaboradores (6 do front office e 2 do back office) e 4 supervisores ( 2 do front office, 1 do back office e 1 do time de apoio à gestão), vistos pelos representantes como detentores de competências capazes de contribuir com a efetiva execução do projeto.

Ante à necessidade de aumento da capacidade de agir do front office, a sugestão destes pesquisadores, validada pelos participantes-chaves e representantes da empresa, foi transferir a ele certas atividades dos supervisores e do back office, para redução do ciclo de atendimento: aos operadores, a alteração traria enriquecimento à função; aos supervisores, mais tempo dedicado à gestão de pessoas; aos analistas, o novo papel de gerir, e, não mais, processar, solicitações de tratamento por exceção; aos clientes, maior agilidade.

Ao longo de 3 semanas, estes pesquisadores envolveram os participantes-chaves no desenvolvimento de um roteiro (Figura 2), baseado no modelo teórico proposto, através do qual se pudesse gerir a instituição desta nova ideia - Projeto Agir - na empresa.

Figura 2: Roteiro para aplicação do modelo teórico proposto

\begin{tabular}{|c|c|c|c|c|}
\hline \multirow{3}{*}{ 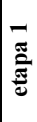 } & função & aspecto & dimensão & processo básico \\
\hline & planejamento & negócio & visão compartilhada & obtenção de conhecimento \\
\hline & \multicolumn{2}{|c|}{ diretrizes } & definir o propósito do "projeto agir" & definir o funcionamento do "projeto agir" \\
\hline \multirow{3}{*}{ 紊 } & função & aspecto & dimensão & processo básico \\
\hline & organização & processos & estrutura organizacional & organização do conhecimento \\
\hline & \multicolumn{2}{|c|}{ diretrizes } & identificar as responsabilidades & identificar atividades transferiveis ao front office \\
\hline \multirow{3}{*}{ 常 } & função & aspecto & dimensão & processo básico \\
\hline & direção & tecnologia & aprendizado & difusão do conhecimento \\
\hline & \multicolumn{2}{|c|}{ diretrizes } & definir os mecanismos de capacitação & definir a metodologia de capacitação \\
\hline \multirow{3}{*}{ 齐 } & função & aspecto & dimensão & processo básico \\
\hline & controle & pessoas & reconhecimento & exploração do conhecimento \\
\hline & \multicolumn{2}{|c|}{ diretrizes } & definir os mecanismos de monitoração do desempenho & definir indicadores de desempenho e recompensa \\
\hline
\end{tabular}

Fonte: Elaborado pelos autores

Com etapas interdependentes, o roteiro fornecia diretrizes que iam da conscientização das pessoas ao uso do saber coletivo, unindo (vide 2.1) funções administrativas, aspectos essenciais à ação, dimensões do empowerment e processos básicos de gestão do conhecimento.

\subsection{Objetivo intermediário 4 - uso do roteiro para aplicação do modelo teórico proposto}

Ao longo de 30 semanas, pesquisadores e participantes-chaves, atuando em conjunto, seguiram as diretrizes relativas a cada uma das etapas do roteiro para aplicação do modelo teórico proposto, visando implementar o Projeto Agir no customer care:

- na definição do propósito do projeto, criou-se um programa de comunicação multimídia, cuja tônica foi uma relação direta (sem intermediários), dinâmica (estimulando e aplicando o 
potencial dos funcionários) e única (com soluções específicas) com os clientes: no front office, onde focou-se o enriquecimento da função, o principal desafio foi fazer ver aos operadores que, juntamente com o aumento da responsabilidade, viriam ações de reconhecimento do desempenho; com os supervisores, com quem foi enfatizada a liberação de atividades operacionais e a conquista de maior tempo à gestão de pessoas, o principal esforço foi mostrar que isto não significava "tirar seu poder", mas permitir-lhes cumprir a parte mais importante de seu papel; no back office, onde foi trabalhado seu novo papel gestão de solicitações de tratamento por exceção, o principal cuidado foi garantir que o eventual downsizing da área em função da eliminação de atividades operacionais não traria demissões, mas oportunidades de realocação; nos times de apoio à gestão, com quem foram explorados os potenciais benefícios do projeto para clientes e colaboradores, a principal preocupação foi a de reforçar seu papel como sendo fundamental na viabilização desta idéia;

- na definição do funcionamento do projeto, solicitações de clientes tratadas por supervisores e back office passaram a ser periodicamente analisadas (os representantes da empresa não autorizaram a divulgação da periodicidade das análises), e, quando superiores a dado volume (os representantes da empresa não autorizaram a divulgação do volume), passavam a ser tratadas pelo front office, capacitado e monitorado;

- na identificação das responsabilidades, a decisão sobre quais solicitações o front office trataria coube ao back office, que também ficou responsável por monitorar seu tratamento pelo front office, e enviar relatórios periódicos para os supervisores e para a gerência;

- na identificação das atividades transferíveis ao front office, foram utilizadas Curvas ABC (i.e. representações gráficas dos tipos e/ou causas de problemas segundo seu grau de importância identificadas por meio de seu grau de frequência - em ordem decrescente, com a categoria maior à esquerda) (MIGUEL, 2001), através das quais se definiu, com base nas atividades de maior volume realizadas pelos supervisores e pelo back office, quais passariam a ser realizadas pelo front office: foram transferidas para o front office doze solicitações de clientes, que, somadas, representavam $35 \%$ das atividades realizadas pelos supervisores e $42 \%$ das atividades realizadas pelo back office (os representantes da empresa não autorizaram a divulgação de quais atividades);

- na definição dos mecanismos de capacitação, a equipe de treinamento do customer care ficou responsável pela qualificação e os supervisores pela reinstrução e/ou encorajamento dos operadores: num total de 20 horas por participante, ao longo de 2 semanas, a qualificação, da qual participaram os supervisores, ocorreu duas horas antes ou duas horas depois dos turnos dos operadores, conforme trabalhassem das $14 \mathrm{~h}-20 \mathrm{~h}$ ou das $08 \mathrm{~h}-14 \mathrm{~h}$, respectivamente (neste processo, a equipe de treinamento do customer care contou com o apoio do back office não só na identificação dos tópicos de ensino, mas também na condução das aulas, como forma de integrar e motivar os envolvidos); contínua e constante, a reinstrução e/ou encorajamento pelos supervisores 
acontecia com base nas monitorações das ligações realizadas por eles próprios e pela equipe controle de qualidade, e nas monitorações pelo back office das solicitações tratadas pelos operadores;

- na definição da metodologia de capacitação, trabalhou-se pela criação de um contexto que não só fornecesse know-how, mas também know-why ao front office, por meio da substituição dos fixed scripts - roteiros fixos que informam aos operadores o que falar e como agir com clientes (BERGEVIN, 2005) - por dynamic scripts - roteiros dinâmicos que lhes fornecem parâmetros de decisão e sugestões de interação (BERGEVIN, 2005): estes dynamic scripts (os representantes da empresa não autorizaram a divulgação de sua estrutura e conteúdo), elaborados com base em entrevistas com supervisores e analistas, nas quais se buscou conhecer como eles decidiam sobre as atividades que passariam a ser realizadas pelos operadores, foram usados na capacitação dos operadores em duas fases: primeiro, entendimento dos dynamic scripts, na formação do conhecimento declarativo (i.e. representação descritiva que apresenta os fatos do mundo, o quê as coisas são e como elas se associam; a descrição de como os objetos são e como se relacionam no mundo, através dos atributos e relacionamentos entre objetos) (ANDERSON, 1996); depois, aplicação dirigida dos dynamic scripts em exercícios, na formação do conhecimento procedural (i.e. percepção automática da forma como as coisas trabalham sob diferentes tipos de circunstâncias, resultante da execução rotineira do conhecimento declarativo, que possibilita a associação entre eventuais problemas e prováveis soluções) (ANDERSON, 1996)

- na definição dos mecanismos de monitoração do desempenho, inicialmente, o back office conferia todas as solicitações tratadas pelo front office, e, pouco a pouco, passou a conferir amostralmente os tratamentos realizados por cada operador(a): esta conferência amostral foi atrelada ao desempenho de cada operador(a) - caso ele(a) executasse suas atividades sem erro, dentro das especificações, a quantidade de inspeções seria mínima, caso fosse detectado erro, ele(a) voltaria à quantidade máxima de inspeções (os representantes da empresa não autorizaram a divulgação das quantidades mínimas e máximas de inspeções);

- na definição dos indicadores de desempenho e recompensa, ante a importância operacional e mercadológica do projeto, os resultados obtidos pelos operadores passaram a contar de modo especial no programa de participação de lucros, já existente na empresa (os representantes da empresa não autorizaram a divulgação do funcionamento deste programa).

\subsection{Objetivo intermediário 5 - avaliação do resultado das ações implementadas}

O público-alvo deste estudo era o front office do customer care, mas, ante o número de pessoas envolvidas (vide 2.2) e a sensibilidade da proposta para o negócio da empresa (vide 2.3), optou-se pelo uso do roteiro para aplicação do modelo teórico proposto na condução de um projeto 
piloto, i.e. aquele no qual conceitos e teorias são testados em pequena escala, sob condições que procuram assemelhar-se a condições reais e normais, com o objetivo de verificar se podem ser empregados e/ou explorados de modo útil (HERZNER, 2009; HORINE, 2009). Assim, de modo aleatório simples (GIL, 1989), obteve-se uma amostra da pesquisa - uma equipe do turno 08h-14h e uma do 14h-20h (períodos de maior volume de ligações) tiveram os números a elas atribuídos sorteados ao acaso, usando-se uma tábua de números aleatórios (GIL, 1989): a opção por duas equipes, além da busca por reduzir a ocorrência do efeito Hawthorne, foi feita visando superar a dificuldade comum de concentração de operadores mais experientes em um determinado turno, em geral, de manhã; a opção por apenas equipes que atuavam no período de maior volume de ligações foi feita com o objetivo de aplicar o modelo teórico proposto num ambiente de teste o mais fiel possível à realidade do customer care.

Definida a amostra (50 operadores), foi analisado o efeito da aplicação do modelo teórico proposto sobre sua capacidade de agir, sobre seu nível de empowerment e sobre a satisfação de seus clientes, bem como o papel da gestão do conhecimento organizacional neste processo.

\subsubsection{Capacidade de agir dos operadores}

Para análise do efeito da aplicação do modelo teórico proposto sobre a capacidade de agir dos operadores no processamento das atividades transferidas dos supervisores e do back office, pesquisadores e participantes-chaves adotaram indicadores já em uso no front office:

- na avaliação do desempenho quantitativo dos operadores, foram utilizados dois indicadores - o indicador "tempo médio de atendimento" (medido em segundos, e obtido dividindo-se o total de tempo gasto pelos operadores no atendimento aos clientes durante suas jornadas de trabalho pelo total de ligações por eles atendidas neste mesmo período) e o indicador "tempo médio disponível" (medido em minutos, e obtido dividindo-se o total de tempo que os operadores estiveram disponíveis para atender aos clientes pelo tempo total de suas jornadas de trabalho, excluindo-se as pausas obrigatórias, previstas em lei);

- na avaliação do desempenho qualitativo dos operadores, foi utilizado o indicador "processos liberados", obtido dividindo-se o total de atividades tratadas dentro dos padrões da empresa pelo total de atividades tratadas.

Após o período de qualificação das equipes que constituíram a amostra desta pesquisa, estes indicadores foram utilizados para acompanhamento de sua capacidade de agir ao longo de 26 semanas.

\subsubsection{Análise do desempenho quantitativo}

Com relação ao indicador "tempo médio de atendimento" (Figura 3), percebe-se que, 
comparado com a média histórica das equipes, antes da aplicação do modelo teórico proposto (174”), o desempenho final dos operadores (191”) piorou em 10\%.

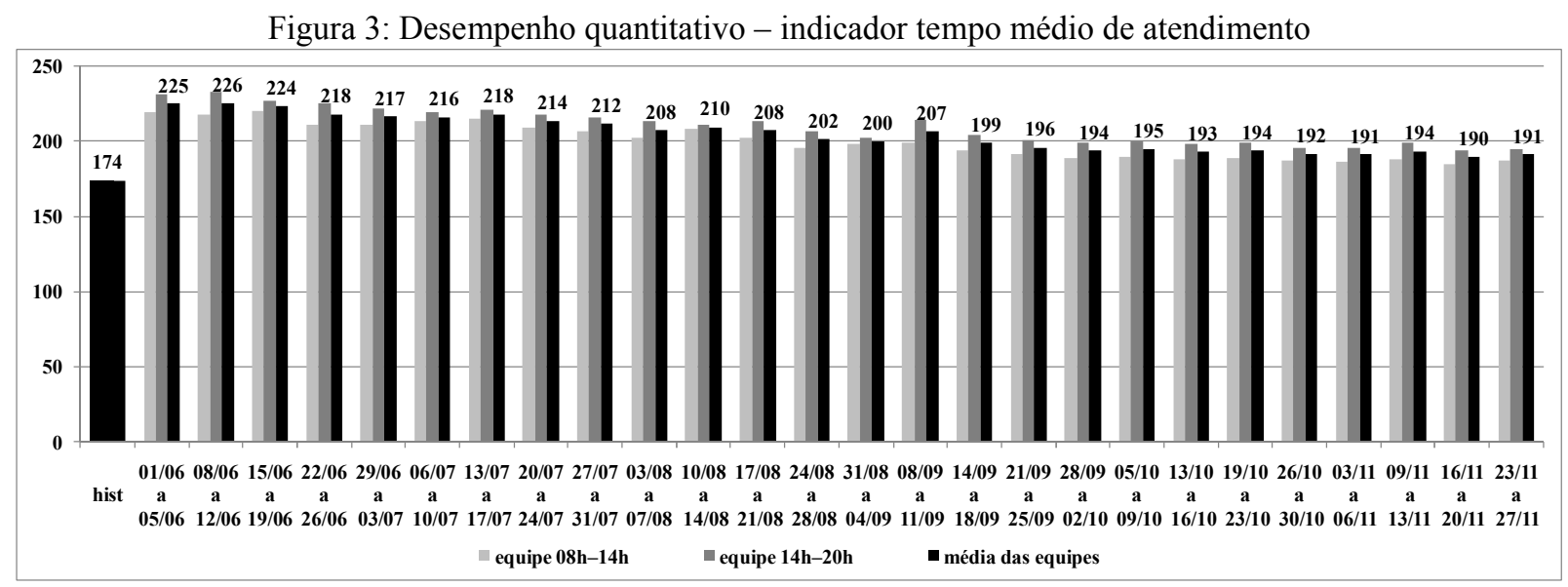

Fonte: Elaborado pelos autores

Contudo, embora gerando aumento de custo no front office, este resultado possibilitou o downsizing no back office, implicando, segundo os representantes da empresa, significativa redução de custo, propiciando vantagem financeira para o customer care como um todo (os representantes da empresa não autorizaram a divulgação destes números).

Com relação ao indicador "tempo médio disponível" (Figura 4), percebe-se que, comparado com a média histórica das equipes, antes da aplicação do modelo teórico proposto (323'), o desempenho final dos operadores (327') melhorou em 1,24\%.

Figura 4: Desempenho quantitativo - indicador tempo médio disponível

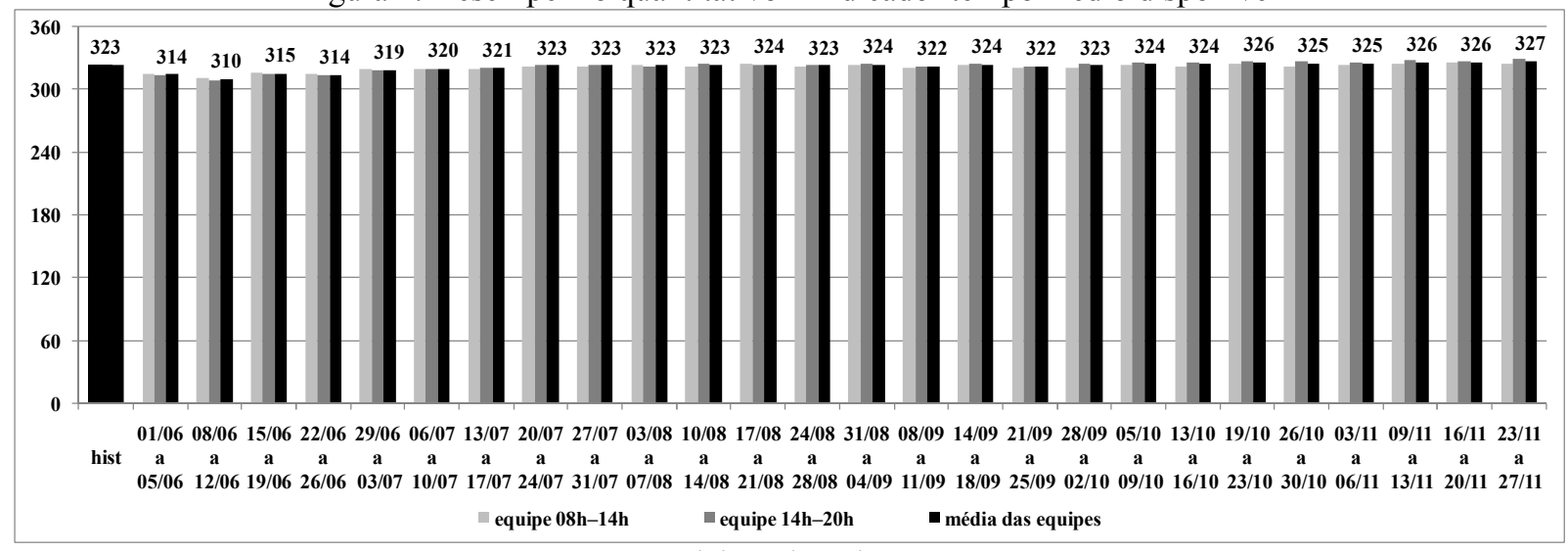

Fonte: Elaborado pelos autores

Segundo os representantes da empresa este resultado foi muito bom, quando considerado que o desempenho histórico do front office estava abaixo da meta definida (330'), indicando maior nível de comprometimento dos operadores, após a aplicação do modelo teórico proposto.

\subsubsection{Análise do desempenho qualitativo}

Com relação ao indicador "processos liberados" (Figura 5), percebe-se que, o desempenho 
final dos operadores $(99,98 \%)$ alcançou, de modo oscilante, contudo, a média histórica do atingida pelo back office e pelos supervisores, antes da aplicação do modelo teórico proposto (99,98\%).

Figura 5: Desempenho qualitativo - processos liberados

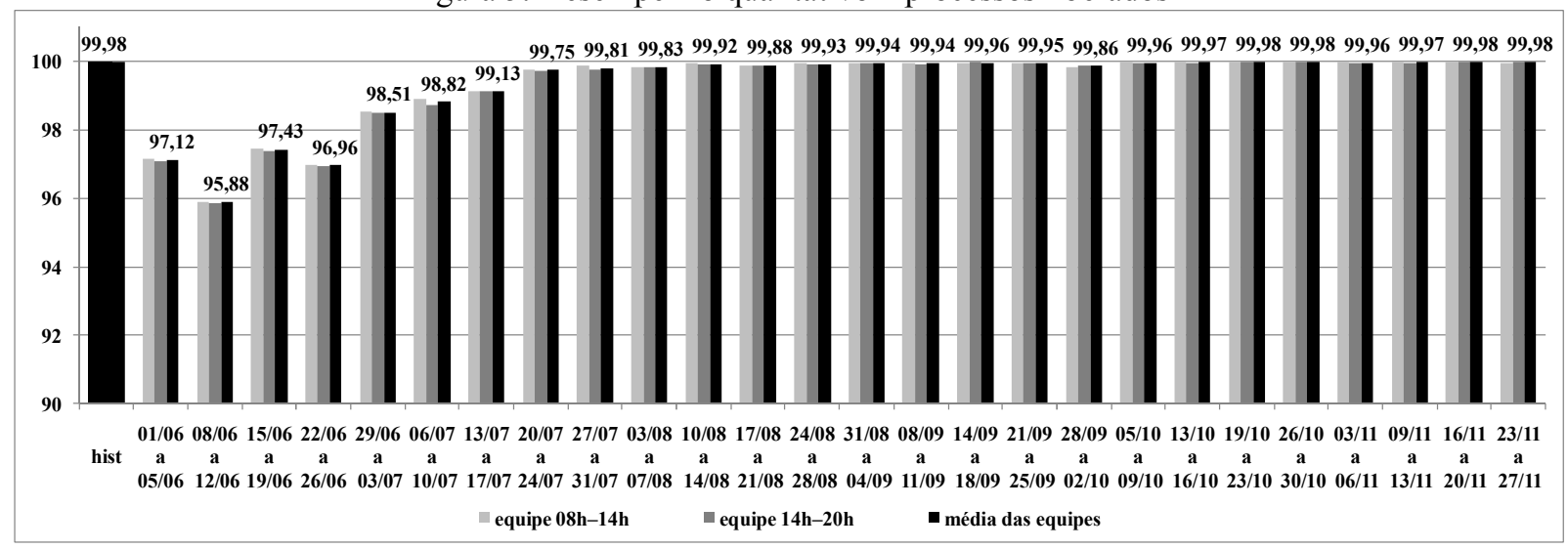

Fonte: Elaborado pelos autores

Apesar de ainda não estabilizado, o resultado agradou aos representantes da empresa, que justificaram sua satisfação por levarem em conta a incorporação de uma "nova, complexa e sensível atividade pelos operadores", e por ser este "o início de uma curva de aprendizagem".

\subsubsection{Nível de empowerment dos operadores}

Para análise do efeito da aplicação do modelo teórico proposto sobre o nível de empowerment dos operadores, pesquisadores e participantes-chaves criaram um questionário eletrônico, usado ao longo de 1 semana no início e 1 semana no fim do estudo - os operadores, após explicação dos participantes-chaves nas reuniões semanais de equipe, responderam a questões fechadas e de estimação (vide 2), que buscaram sua percepção (Figura 6) quanto à existência e clareza das dimensões do empowerment (HERRENKOHL et al.,1999) (vide 2.1).

Figura 6: Nível de empowerment dos operadores

\begin{tabular}{|c|c|c|c|c|c|c|c|c|c|c|c|c|c|c|}
\hline \multirow{3}{*}{$\begin{array}{l}\text { dimensões fundamentais } \\
\text { do empowerment }\end{array}$} & \multicolumn{7}{|c|}{ respostas obtidas de 11 a 15/05 (início do estudo) } & \multicolumn{7}{|c|}{ respostas obtidas de 23 a 27/11 (fim do estudo) } \\
\hline & \multicolumn{2}{|c|}{ existem } & \multicolumn{2}{|c|}{ 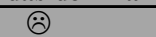 } & \multicolumn{3}{|c|}{ são claras } & \multicolumn{2}{|c|}{ existem } & \multicolumn{2}{|c|}{ () } & \multicolumn{2}{|c|}{ ão claras } & (;) \\
\hline & $\operatorname{sim}$ & não & 1 & 2 & 3 & 4 & 5 & $\operatorname{sim}$ & não & 1 & 2 & 3 & 4 & 5 \\
\hline \multirow{2}{*}{ visão compartilhada } & 8 & 42 & 25 & 15 & 4 & 3 & 3 & 45 & 5 & 1 & 4 & 4 & 17 & 24 \\
\hline & $16 \%$ & $84 \%$ & $50 \%$ & $30 \%$ & $8 \%$ & $6 \%$ & $6 \%$ & $90 \%$ & $10 \%$ & $2 \%$ & $8 \%$ & $8 \%$ & $34 \%$ & $48 \%$ \\
\hline \multirow{2}{*}{ estrutura organizacional } & 11 & 39 & 25 & 13 & 10 & 1 & 1 & 43 & 7 & 1 & 3 & 8 & 12 & 26 \\
\hline & $22 \%$ & $78 \%$ & $50 \%$ & $26 \%$ & $20 \%$ & $2 \%$ & $2 \%$ & $86 \%$ & $14 \%$ & $2 \%$ & $6 \%$ & $16 \%$ & $24 \%$ & $52 \%$ \\
\hline \multirow{2}{*}{ aprendizado } & 12 & 38 & 21 & 18 & 9 & 1 & 1 & 45 & 5 & 1 & 6 & 7 & 17 & 19 \\
\hline & $24 \%$ & $76 \%$ & $42 \%$ & $36 \%$ & $18 \%$ & $2 \%$ & $2 \%$ & $90 \%$ & $10 \%$ & $2 \%$ & $12 \%$ & $14 \%$ & $34 \%$ & $38 \%$ \\
\hline \multirow{2}{*}{ reconhecimento } & 7 & 43 & 23 & 18 & 7 & 1 & 1 & 47 & 3 & 1 & 1 & 7 & 18 & 23 \\
\hline & $14 \%$ & $86 \%$ & $46 \%$ & $36 \%$ & $14 \%$ & $2 \%$ & $2 \%$ & $94 \%$ & $6 \%$ & $2 \%$ & $2 \%$ & $14 \%$ & $36 \%$ & $46 \%$ \\
\hline média & $19 \%$ & $81 \%$ & $47 \%$ & $32 \%$ & $15 \%$ & $3 \%$ & $3 \%$ & $90 \%$ & $10 \%$ & $2 \%$ & $7 \%$ & $13 \%$ & $32 \%$ & $46 \%$ \\
\hline
\end{tabular}

Fonte: Elaborado pelos autores, com base em HERRENKOHL et al. (1999)

Analisando-se as respostas obtidas no início do estudo, percebe-se que, em média, 19\% dos operadores - respostas "sim" - acreditavam que as dimensões fundamentais do empowerment eram parte de seu dia-a-dia, e que, em média, $81 \%$ deles achavam que elas não eram claras - respostas "1" (discordo totalmente) e "2" (discordo parcialmente); analisando-se as respostas obtidas no fim 
do estudo, percebe-se que, em média, 90\% dos operadores - respostas "sim" - acreditavam que as dimensões fundamentais do empowerment eram parte de seu dia-a-dia, e que, em média, 78\% deles, achavam que elas eram claras - "4" (concordo parcialmente) e "5" (concordo totalmente): quando estas percepções dos operadores são combinadas com os resultados das análises de seu desempenho quantitativo e qualitativo, é possível inferir que a aplicação do modelo teórico proposto contribuiu com o aumento de seu nível de empowerment.

\subsubsection{Satisfação dos clientes}

Para análise do efeito da aplicação do modelo teórico proposto sobre a satisfação dos clientes atendidos pelos operadores que constituíram a amostra deste estudo, pesquisadores e participantes-chaves recorreram à equipe de controle de qualidade (vide 2.2): utilizando os mesmos critérios de suas pesquisas de satisfação dos clientes atendidos pelo front office (os representantes da empresa não autorizaram a divulgação do funcionamento deste programa), ao longo de 26 semanas, os monitores, diariamente, ligavam para clientes atendidos pelas duas equipes, aleatoriamente escolhidos, e registravam suas entrevistas (Figura 7).

Figura 7: Satisfação dos clientes

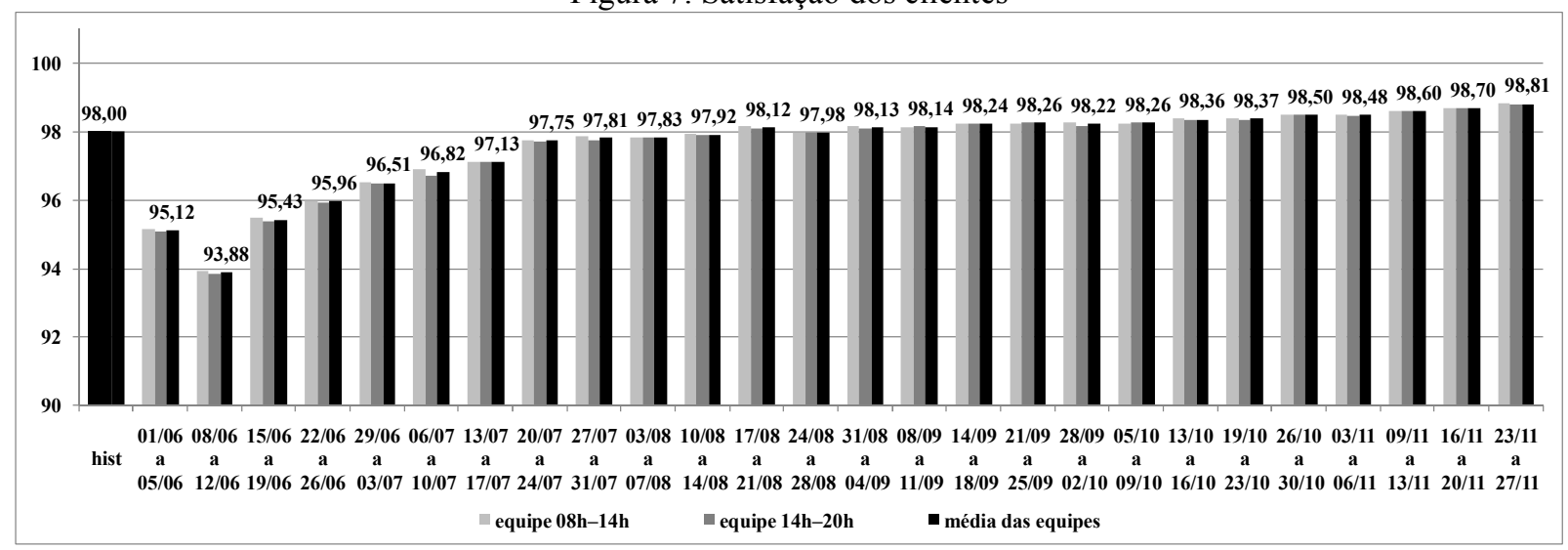

Fonte: Elaborado pelos autores

Percebe-se que, comparado com a média histórica do front office, antes da aplicação do modelo teórico proposto (98\%), o nível de satisfação dos clientes, numa tendência ascendente, melhorou $0,83 \%$ ao final da pesquisa $\left(98,81^{\prime}\right)$.

\subsubsection{Papel da gestão do conhecimento}

Para análise do papel da gestão do conhecimento na aplicação do modelo teórico proposto, pesquisadores e participantes-chaves criaram um questionário eletrônico, usado ao longo de 1 semana no início e 1 semana no fim do estudo - os operadores, após explicação dos participanteschaves nas reuniões semanais de equipe, responderam a questões fechadas e de estimação (vide 2), que buscaram sua percepção (Figura 8) quanto à existência e clareza aos processos básicos 
associados à gestão do conhecimento empresarial (SKYRME, 1999) (vide 2.1).

Figura 8: Papel da gestão do conhecimento

\begin{tabular}{|c|c|c|c|c|c|c|c|c|c|c|c|c|c|c|}
\hline \multirow{3}{*}{$\begin{array}{l}\text { processos básicos de } \\
\text { ges tão do conhecimento }\end{array}$} & \multicolumn{7}{|c|}{ respostas obtidas de 11 a 15/05 (início do estudo) } & \multicolumn{7}{|c|}{ respostas obtidas de 23 a $27 / 11$ (fim do estudo) } \\
\hline & \multicolumn{2}{|c|}{ existem } & \multicolumn{2}{|l|}{ (2) } & \multicolumn{3}{|c|}{ ão claras } & \multicolumn{2}{|c|}{ existem } & \multicolumn{2}{|l|}{ :) } & \multicolumn{2}{|c|}{ são claras } & \multirow{2}{*}{$\begin{array}{c};) \\
5\end{array}$} \\
\hline & sim & não & 1 & 2 & 3 & 4 & 5 & sim & não & 1 & 2 & 3 & 4 & \\
\hline \multirow{2}{*}{ obtenção } & 7 & 43 & 26 & 18 & 3 & 2 & 1 & 45 & 5 & 2 & 1 & 5 & 15 & 27 \\
\hline & $14 \%$ & $86 \%$ & $52 \%$ & $36 \%$ & $6 \%$ & $4 \%$ & $2 \%$ & $90 \%$ & $10 \%$ & $4 \%$ & $2 \%$ & $10 \%$ & $30 \%$ & $54 \%$ \\
\hline \multirow{2}{*}{ organização } & 10 & 40 & 26 & 15 & 8 & 1 & 0 & 43 & 7 & 0 & 1 & 10 & 13 & 26 \\
\hline & $20 \%$ & $80 \%$ & $52 \%$ & $30 \%$ & $16 \%$ & $2 \%$ & $0 \%$ & $86 \%$ & $14 \%$ & $0 \%$ & $2 \%$ & $20 \%$ & $26 \%$ & $52 \%$ \\
\hline \multirow{2}{*}{ difusão } & 11 & 39 & 24 & 19 & 5 & 1 & 1 & 45 & 5 & 1 & 1 & 9 & 17 & 22 \\
\hline & $22 \%$ & $78 \%$ & $48 \%$ & $38 \%$ & $10 \%$ & $2 \%$ & $2 \%$ & $90 \%$ & $10 \%$ & $2 \%$ & $2 \%$ & $18 \%$ & $34 \%$ & $44 \%$ \\
\hline \multirow{2}{*}{ exploração } & 6 & 44 & 24 & 20 & 6 & 0 & 0 & 47 & 3 & 1 & 1 & 6 & 17 & 25 \\
\hline & $12 \%$ & $88 \%$ & $48 \%$ & $40 \%$ & $12 \%$ & $0 \%$ & $0 \%$ & $94 \%$ & $6 \%$ & $2 \%$ & $2 \%$ & $12 \%$ & $34 \%$ & $50 \%$ \\
\hline média & $17 \%$ & $83 \%$ & $50 \%$ & $36 \%$ & $11 \%$ & $2 \%$ & $1 \%$ & $90 \%$ & $10 \%$ & $2 \%$ & $2 \%$ & $15 \%$ & $31 \%$ & $50 \%$ \\
\hline
\end{tabular}

Fonte: Elaborado pelos autores, com base em SKYRME (1999)

Analisando-se as respostas obtidas no início do estudo, percebe-se que, em média, 17\% dos operadores - respostas "sim" - acreditavam que os processos básicos associados à gestão do conhecimento empresarial eram parte de seu dia-a-dia, e que, em média, $86 \%$ deles não os achavam claros - respostas "1" (discordo totalmente) e "2" (discordo parcialmente); analisando-se as respostas obtidas no fim do estudo, percebe-se que, em média, 90\% dos operadores - respostas “sim" - acreditavam que os processos básicos associados à gestão do conhecimento empresarial eram parte de seu dia-a-dia, e que, em média, 81\% deles, achavam-nos claros - "4" (concordo parcialmente) e " 5 " (concordo totalmente).

\section{Conclusão}

Considerando a melhoria no desempenho quantitativo (vide 2.5.1.1) e qualitativo (vide 2.5.1.2) dos operadores, o aumento do nível de satisfação dos clientes (vide 2.5.3), e a percepção dos operadores quanto à melhoria de seu nível de empowerment (vide 2.5.2) e dos processos de gestão do conhecimento empresarial (vide 2.5.4), é possível afirmar - sem, contudo, qualquer pretensão de generalização - que a resposta para a pergunta de pesquisa deste estudo é "Sim, a gestão do conhecimento pode contribuir para uma efetiva implementação da abordagem empowerment em contact centers".

\section{Considerações Finais}

Os contact centers vêm se desenvolvendo em ritmo incrivelmente forte, ocupando, hoje, o segundo lugar no ranking de empregadores privados no Brasil (DAMASCENO, 2005) e contribuindo com $7 \%$ do total dos postos de trabalho oferecidos em todo o mundo (BERGEVIN, 2005); apesar disso, na realização deste trabalho, observou-se limitada existência de estudos científicos e amplos desafios enfrentados por empresários, gestores e funcionários no setor, indicando a necessidade/oportunidade de maior atenção por parte da comunidade acadêmica. 


\begin{abstract}
This paper aimed at developing and testing a proposal based on knowledge management to enable a contact center operation to harvest the benefits of workforce empowerment while minimizing the risks of such a managerial approach. In an experimental, interventionist research, flavored with a critical orientation, the case of such an operation in São Paulo was studied. In light of the findings, it is possible to conclude, without any room for generalization, however, that knowledge management can contribute to an effective implementation of the empowerment approach in contact centers.
\end{abstract}

Key words: knowledge management; empowerment; contact center; operations.

\title{
Referências
}

ALSOP, R.; BERTELSEN, M.; HOLLAND, J. Empowerment in practice: from analysis to implementation. Washington: World Bank, 2006.

ALVARENGA NETO, R. C. D. Gestão do conhecimento em organizações. São Paulo: Saraiva, 2008.

ANDERSON, J. R. ACT: A simple theory of complex cognition. American psychologist, Washington, v. 51, 1996.

ARGYRIS, C. Empowerment: the emperor's new clothes. Harvard Business Review, Boston, May/Jun; 1998.

BATESON, J. E. G.; HOFFMAN, K. D. Marketing de serviços. Porto Alegre: Bookman, 2001.

BERGEVIN, R. Call centers for dummies. Mississauga: Wiley, 2005.

BIAZZI, R. Lei que regulamenta regras para call centers completa um ano. Jornal Hoje. São Paulo, dez. 2009. Disponível em: < http://g1.globo.com/jornalhoje>. Acesso em: 08 dez. 2009.

BLANCHARD, K.; CARLOS, J. P.; RANDOLPH, A. The 3 keys to empowerment: release the power within people for astonishing results. Sydney: Berrett-Koehler, 2001.

CARBONE, P. P. et al. Gestão por competências e gestão do conhecimento. Rio de Janeiro: FGV, 2006.

COLE, N. How employee empowerment improves manufacturing performance. The Academy of Management Executive, Detroit, Feb., 1995.

COLLINS, J. C.; PORRAS, J. I. Construindo a visão da empresa. HSM Management, Barueri, mar./abr., 1998.

CORRÊA, H. L.; CAON, M. Gestão de serviços: lucratividade por meio de operações e satisfação dos clientes. São Paulo: Atlas, 2002.

CUNNINGHAM, I.; HYMAN, J. The poverty of empowerment: a critical case study. Personal Review. New York, v.28, 1999.

CURRY, J. The customer marketing method. New York: The Free Press, 2000.

DAFT, R. L. Management. London: Thomson Learning, 2007.

DAMASCENO, S. Todas as posições ocupadas: até logo. Teletime, São Paulo, maio, 2005.

DAVEL, E.; ALCADIPANI, R. Estudos críticos em administração. RAE, São Paulo, v. 43, 2003.

DAVENPORT, T. H. Thinking for a living. Boston: Harvard Business School Press, 2005.

DAVENPORT, T. H.; PRUSAK, L. Working Knowledge. Boston: Harvard Business School Press, 1998.

DAVENPORT, T. H. ; PROBST, G. J. B.; VON PIERER, H. Knowledge management case book. New York: John Wiley Trade, 2002. 
DESSLER, G. How to earn your employees commitment. The Academy of Management Executive, Detroit, May, 1999.

DIJULIUS, J. R. What's the secret: to providing a world-class customer experience. Mississauga: Wiley, 2008.

FIGUEIREDO, S. P. Gestão do conhecimento. Rio de Janeiro: Qualitymark, 2005.

FITZSIMMONS, J.; FITZSIMMONS, M. Service management: operations, strategy, information technology. New York: McGraw Hill, 2007.

GALBRAITH, J. R.; LAWLER, E. E. Organizando para competir no futuro. São Paulo: Makron Books, 1995.

GIL, A. A. C. Métodos e técnicas de pesquisa social. São Paulo: Atlas, 1989.

GINNODO, B. Empowerment: more than a fad. Tapping the network journal, Chicago, Summer, 1992.

HERRENKOHL, R. C.; JUDSON, G. T.; e HEFFNER, J. A. - Defining and measuring empowerment. Journal of Applied Behavioral Science, London, Sep, 1999.

HERZNER, H. Project management: a systems approach to planning, scheduling, and controlling. New York: John Wiley Trade, 2009.

HICKEY, K.; LAKE, N. The customer service handbook. Burlington: Butterworth-Heineman, 2003.

HORINE, G. An absolute beginner's guide to project management. Vancouver: Que Publishing, 2009.

IVES, B.; GERSTING, A. Implementing knowledge management: lessons learned. Inside Knowledge, New York, Feb. 1998. Disponível em: <http://www.ac.com/knowledge management.html>. Acesso em: 30 fev. 2009.

JAFFE, D. T.; SCOTT, C. D. Empowerment: building a committed workforce. London: Kogan Page, 1992.

JAMIL, G. L. Gestão de informação e do conhecimento em empresas brasileiras. Belo Horizonte: Com Arte, 2006.

JOHNSTON, R.; CLARK, G. Administração de operações de serviço. São Paulo: Atlas, 2002.

KAUFMANN, F. Metodologia das ciências sociais. Rio de Janeiro: Francisco Alves, 1977.

LARA, C. R. D. A atual gestão do conhecimento: a importância de avaliar e identificar o capital. São Paulo: Nobel, 2004.

LAWLER, E. E. Estratégia versus funcionários. HSM Management, São Paulo, set./out., 1998.

LAWLER, E. E. Organizing for high performance. New York: John Wiley Trade, 2001.

LEILBOLD, M.; PROBST, G. J. B.; GIBBERT, M. Strategic management in the knowledge economy: new approaches and business applications. New York: John Wiley Trade, 2005.

LELAND, K. ; BAILEY, K. Customer service for dummies. Mississauga: Wiley, 2006.

LEVITT, T. Production line approach to service. Harvard Business Review, Boston, Sept./Oct, 1972. Industrialization of service. Harvard Business Review, Boston, Sept./Oct., 1976.

LUCAS, R. W. Customer service skills for success. New York: McGraw Hill, 2008.

MADRUGA, R. P. Gestão moderna de call center/telemarketing. São Paulo: Atlas, 2006.

MALONE, T. W. The future of work: how the new order of business will shape your organization, your management style, and your life. Boston, Harvard Business School Press, 2004.

MARCONI, M. A.; LAKATOS, E. M. Técnicas de pesquisa. São Paulo: Atlas, 1990. 
Fundamentos de metodologia científica. São Paulo: Atlas, 2006.

MIGUEL, P. A. C. Qualidade: enfoques e ferramentas. São Paulo: Artliber, 2001.

NONAKA, I. The knowledge creating company. Harvard Business Review, Boston, Nov./Dec., 1991.

NICKERSON, A. G. Not by the seat of my pants: leadership lessons for the call Center supervisor. Bloomington: iUniverse, 2004.

PFEIFFER, I.; DUNLOP, J. Increasing productivity through empowerment. Supervisory management, New York, Jan., 1990.

PONTES, A. Call center: dor de cabeça continua e o único jeito é reclamar! Veja.com, São Paulo, out. 2009. Disponível em: <http://veja.abril.com.br/noticia/brasil>. Acesso em: 08 dez. 2009.

POPPER, K. S. A lógica da pesquisa científica. São Paulo: Cultrix, 1975a.

Conhecimento objetivo. São Paulo: Cultrix, 1975 b.

POTTERFIELD, T. A. The business of employee empowerment: democracy and ideology in the workplace. Abingdon: Greenwood, 1999.

QUINN, R. E.; SPREITZER, G. M. The Road to Empowerment: seven questions every leader should consider. IEEE Engineering Management Review, Macon, Summer, 1999.

SANTIAGO JR., J. R. S. Gestão do conhecimento: a chave para o sucesso empresarial. São Paulo: Novatec, 2004.

SHARP, D. Call center operation. Burlington: Butterworth-Heineman, 2003.

SIMONS, R. Control in an age of empowerment. Boston: Harvard Business School Press, 2008.

SKYRME, D. J. Knowledge networking. London: Butterworth Heinemann, 1999.

SUFFERT, C J. Gestão do conhecimento como solução. Rio de Janeiro: Qualitymark, 2008.

SVEIBY, K. E. The new organizational wealth. Sydney: Berrett-Koehler, 1998.

THIOLLENT, M. Pesquisa-ação nas organizações. São Paulo: Atlas, 1997.

TOMASKO, R. M. Rethinking: repensando as corporações. São Paulo: Macron, 1994.

TRUJILlO FERRARI, A. Metodologia da ciência. Rio de Janeiro: Kennedy, 1974.

VERGARA, S.C. Projetos e relatórios de pesquisa em administração. São Paulo: Atlas, 1998.

Métodos de pesquisa em administração. São Paulo: Atlas, 2005.

VERGARA, S. C.; BRANCO, P. D. Empresa humanizada. RAE, São Paulo, v. 41, 2001.

VIALli, A.; CRUZ, R. Após um ano, empresa ainda descumpre lei do call center. O Estado de São Paulo, São Paulo, dez. 2009. Disponível em: <http://www.estadao.com.br/noticias/economia>. Acesso em: 08 dez. 2009.

WALTON, D. From bad to worse: the shortest route to call Center destruction. Charleston: BookSurge, 2006.

\section{Dados dos autores:}

Nome completo: Samuel Ribeiro Tavares

Filiação institucional: Universidade Nove de Julho - UNINOVE 
Departamento: Diretoria de Ciências Gerenciais

Função ou cargo ocupado: Professor

Endereço completo para correspondência (bairro, cidade, estado, país e CEP): Avenida Francisco Matarazzo, 612. Água Branca, São Paulo - SP. CEP 05001-010

Telefones para contato: 01136080110

e-mail: samueltavares@yahoo.com

Nome completo: Milton Vieira Junior

Filiação institucional: Universidade Nove de Julho - UNINOVE

Departamento: Programa de Pós-graduação em Engenharia de Produção

Função ou cargo ocupado: Professor Pesquisador

Endereço completo para correspondência (bairro, cidade, estado, país e CEP): Avenida Francisco Matarazzo, 612. Água Branca, São Paulo - SP. CEP 05001-010

Telefones para contato: 01136659355

e-mail: mvieirajr@ig.com.br

Enviado em: 29/01/2011

Aprovado em: 03/10/2011 